\title{
ANÁLISIS ESPACIO-TEMPORAL DE INCENDIOS DEL NORTE Y CENTRO DE SURAMÉRICA: 2009-2019
}

\author{
SPATIO-TEMPORAL ANALYSIS OF NORTHERN AND CENTRAL FIRES FROM \\ SOUTH AMERICA: 2009-2019
}

\author{
Brigette López-Guevara \\ Estudiante de ingeniería ambiental, Semillero BioSIG \\ Universidad Nacional Abierta y a Distancia \\ ORCID: 0000-0001-5750-6182 \\ Email: blopezgu@unadvirtual.edu.co
}

\begin{abstract}
Citación: López-Guevara, B. (2021). Análisis espacio-temporal de incendios del norte y centro de Suramérica: 2009-2019. Agricolae \& Habitat, 4(2), 33 - 43. DOI:

https://doi.org/10.22490/26653176.4542
\end{abstract}

\section{RESUMEN}

Contextualización: la frecuencia, poder radiativo y tamaño de los incendios en el mundo se ha incrementado, generando graves impactos sociales, ambientales y económicos.

Vacío del conocimiento: A pesar de ello, se cuenta con una cantidad limitada de investigaciones frente a este problema, especialmente en el norte (NSA) y centro de Suramérica (CSA) que permitan crear políticas públicas con base en la ciencia y tecnología.

Propósito: así las cosas, el presente proyecto busco analizar espacial y temporalmente los incendios del norte y centro de Suramérica entre 2009 y 2019.

Metodología: se utilizó información del espectrorradiómetro de imágenes de media resolución (MODIS) a bordo de los satélites Aqua y Terra de la NASA. Los datos de incendios con una confianza mayor al $75 \%$ fueron seleccionados y procesados en lenguaje de programación $R$. Se identifico espacialmente aquellos incendios con el mayor poder radiativo [MW] (por encima del percentil 90). La distribución del conteo diario de incendios se analizó de forma mensual y anual. 
Resultados y conclusiones: durante los primeros cuatro meses del año, el NSA es afectado por incendios de más de 3000 MW en la cuenca de la Orinoquia; mientras que el CSA es afectado en más de un $90 \%$ por incendios de más $8800 \mathrm{MW}$, principalmente durante agosto, septiembre y octubre.

Palabras clave: gestión ambiental, tecnología espacial, satélite artificial, estadísticas ambientales, gestión de riesgos

\section{ABSTRACT}

Contextualization: the frequency, radiative power, and size of fires in the world has increased, generating serious social, environmental, and economic impacts.

Knowledge gap: Despite this, there is a limited amount of research on this problem, especially in the northern (NSA) and central South America (CSA).

\section{RESUMEN GRÁFICO}

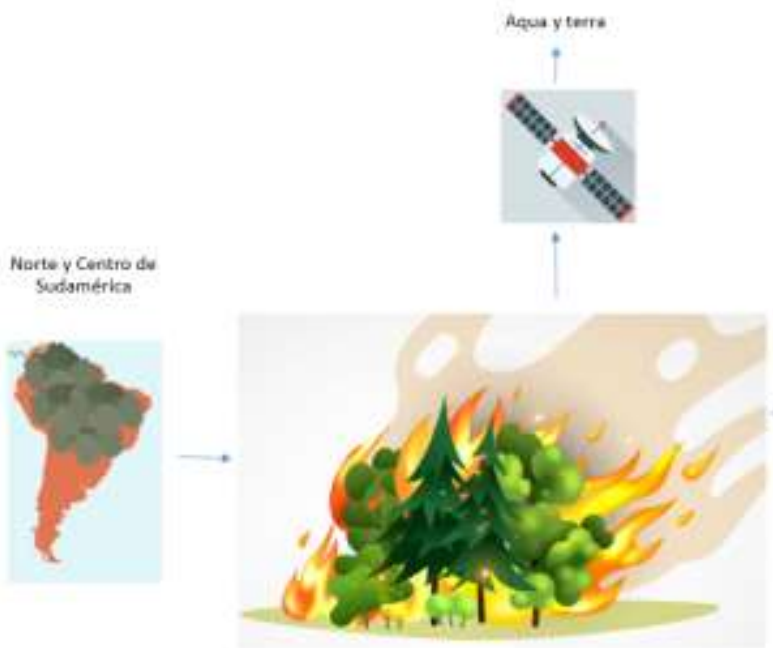

Purpose: this project sought to analyze spatially and temporally the fires in NSA and CSA between 2009 and 2019.

Methodology: information from the medium resolution imaging spectroradiometer (MODIS) on board the Aqua and Terra NASA's satellites was used. The fire data with a confidence greater than $75 \%$ were selected and processed in $\mathrm{R}^{\prime}$ programming. Fires with the highest radiative power [MW] (above the 90 th percentile) were spatially identified. The distribution of daily fire counting was analyzed on a monthly and annual basis.

Results and conclusions: during the first four months of the year, the NSA is affected by fires of more than $3000 \mathrm{MW}$ in the Orinoquia basin; while the CSA is affected in more than $90 \%$ by fires of more than 8,800 MW, mainly during August, September, and October.

Keywords: Environmental management, space technology, artificial satellites, Environmental statistics, risk management
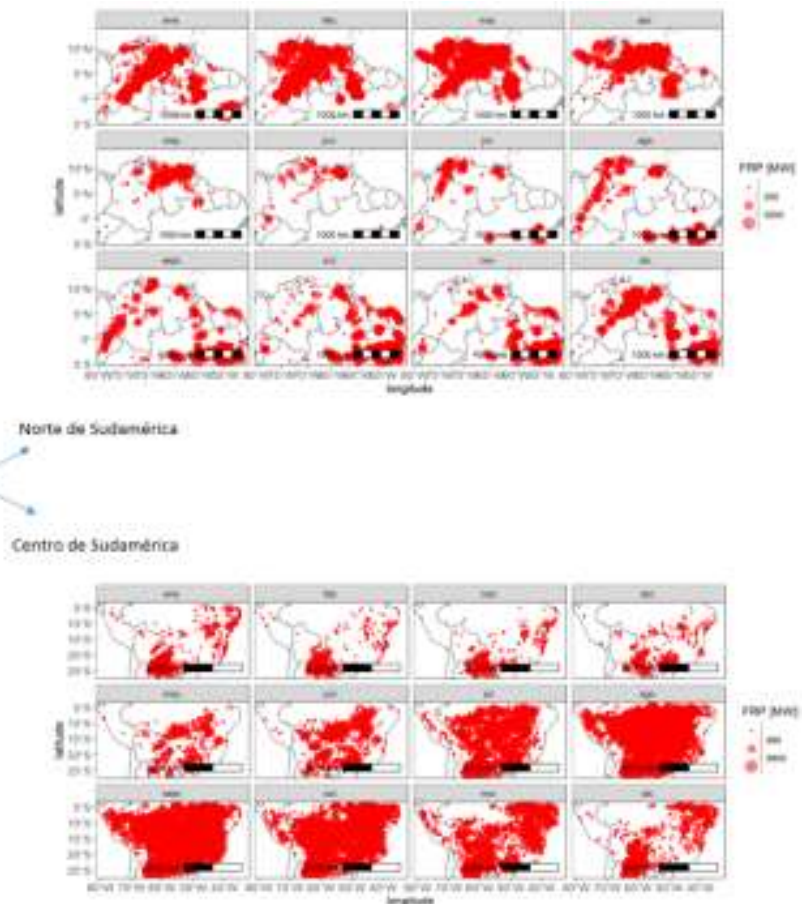

Fuente: autora 


\section{INTRODUCCIÓN}

Müller et al. (2013) afirma "Los incendios juegan un importante rol en la dinámica energética de los ecosistemas como en el manejo de la tierra". Pimm et al.,(1995) afirma "Es reconocido cada vez más el impacto de las actividades humanas sobre los ecosistemas, dando lugar a fuertes modificaciones sobre los patrones espaciales de la biodiversidad y sus servicios a nivel global". Parr \& Chown, (2003) afirma "El fuego ha sido asociado con la "expresión del banco de semillas mediante diversos mecanismos ecológicos".

Müller et al., (2013) afirma "Se ha asociado con alteraciones del microclima ",MORTON et al.,(2008) afirma " y la interacción atmósfera-vegetación". Así mismo, con efectos económicos y sociales a nivel localregional. En Latinoamérica, aunque el "fuego puede ser un mecanismo natural en muchos ecosistemas, las acciones humanas han alterado los regímenes naturales" generando pérdida en servicios ecosistémicos que mantienen el equilibro natural

Marengo et al.,(2008) "afirma En Suramérica, el incremento de incendios recientes está asociado con periodos de sequias", Ray et al.,(2005) afirma "particularmente en la cuenca del Amazonas, dónde se ha asociado este aumento con eventos climáticos extremos como el fenómeno del niñooscilación sur (ENOS)" Marengo et al.,(2008) o con la oscilación del Atlántico Norte. ALENCAR et al (2004) afirma "Los bosques húmedos tropicales de la amazonia fueron gravemente afectados durante las sequías de 1997, 1998, 2005,2007, 2010 y 2015 por grandes incendios, muchos de los cuales se salieron de control desde zonas agrícolas y explotaciones ganaderas". Armenteras et al., (2013) afirma " El incremento de incendios también ha estado asociado a las interacciones entre la deforestación, ocasionada principalmente para el establecimiento de cultivo, en los que el uso del fuego es la herramienta más efectiva para despejar el suelo tanto en el norte como en el centro de Suramérica. Estableciendo un reto a futuro para las presentes $y$ futuras generaciones".

Armenteras et al.,(2013) afirma "La región de norte de Sudamérica y en particular Colombia, Ecuador y Perú, han sufrido un incremento en la frecuencia y extensión de los incendios, sin embargo, han pasado desapercibidos en la literatura. En estos países los incendios ocurren en una amplia variedad de regiones geográficas con condiciones sociales, demográficas, económicas y ambientales especiales. A pesar de ello, no se cuenta con una visión regional del problema, aspecto necesario para formular estrategias de prevención, mitigación y atención. Debido a esto, es fundamental contar con estadísticas sobre incendios para establecer buenas políticas en seguridad contra incendios". Sin embargo, el norte y centro de Suramérica carece de esta herramienta. Si bien existen análisis anuales sobre incendios por parte de La Asociación Internacional para el Estudio de Asuntos Económicos sobre Seguros, "The Geneva Association", y por parte del Comité Técnico Internacional para la Prevención y Extinción de Incendios (CTIF), estos no enfocan sus esfuerzos en las regiones: Norte de Suramérica (NSA) y Centro de Suramérica (CSA). 
A partir del contexto anterior, el presente proyecto busca dar respuesta a la pregunta general: ¿Cuál fue el comportamiento espacio-temporal de los incendios del norte y centro de Suramérica durante los años 20092019?

Considerando que se cuenta con una cantidad limitada de investigaciones que hagan frente a este problema, especialmente en el norte y centro de Suramérica. La presente investigación tiene como objetivo principal analizar espacial y temporalmente los incendios del norte y centro de Suramérica entre 2009-2019. Para su cumplimiento se especificaron los siguientes objetivos específicos:

*Determinar la serie temporal de número de incendios diarios multi-anual y multi-mensual en el norte y centro de Suramérica entre 2009-2019 haciendo uso de del lenguaje de programación $\mathrm{R}$.

*Identificar la distribución espacial de aquellos incendios del norte y centro de Suramérica entre 2009-2019 cuyo poder radiativo $[\mathrm{MW}]$ se encuentra por encima del percentil 90.

\section{MATERIALES Y MÉTODOS}

\section{- Área de estudio}

En esta investigación se considera el norte de Suramérica como el dominio (longitud $=-79.0, \quad$ latitud $=-4.4 \quad$ como esquina inferior izquierda; longitud $=-51.7$, latitud = 13.1 como esquina superior derecha) (Figura 1a). El centro de Suramérica se consideró como el dominio (longitud $=-79.0$, latitud $=-26.5$ como esquina inferior izquierda; $\quad$ longitud $=-34.2$, latitud $=-4.4$ como esquina superior derecha) (Figura 1b).

Armenteras et al., (Armenteras et al., 2013) afirma Dentro de los ecosistemas susceptibles a los incendios, encontramos aquellos que presentan una densidad vegetal considerable además de factores climáticos como baja humedad, gran radiación solar y/o altas temperaturas las cuales pueden generar el comienzo y expansión del fuego. Algunos de los ecosistemas sensibles al fuego son bosques secos, andinos, páramos y el matorral espinoso, los cuales han sufrido transformaciones a tal punto que "presentan procesos extensos de sabanización, paralización, aridización y desertización". 
(a.)

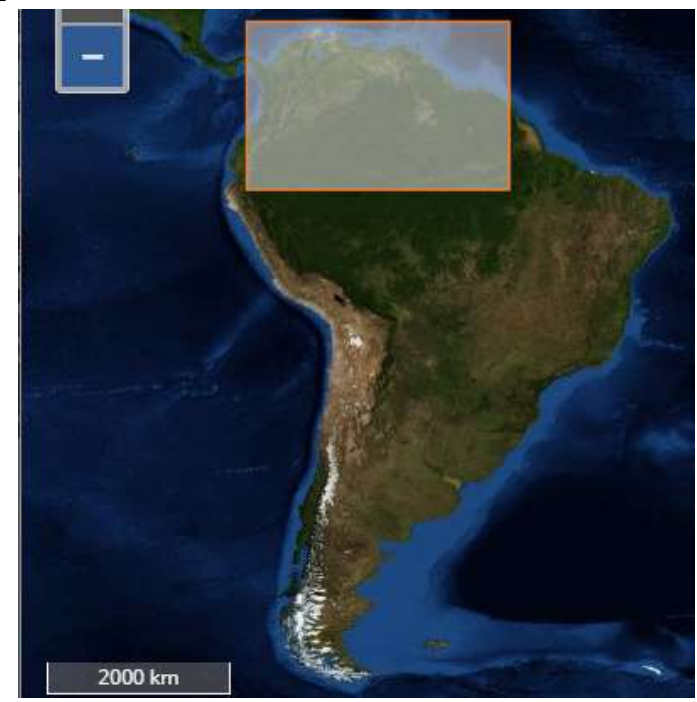

(b.)

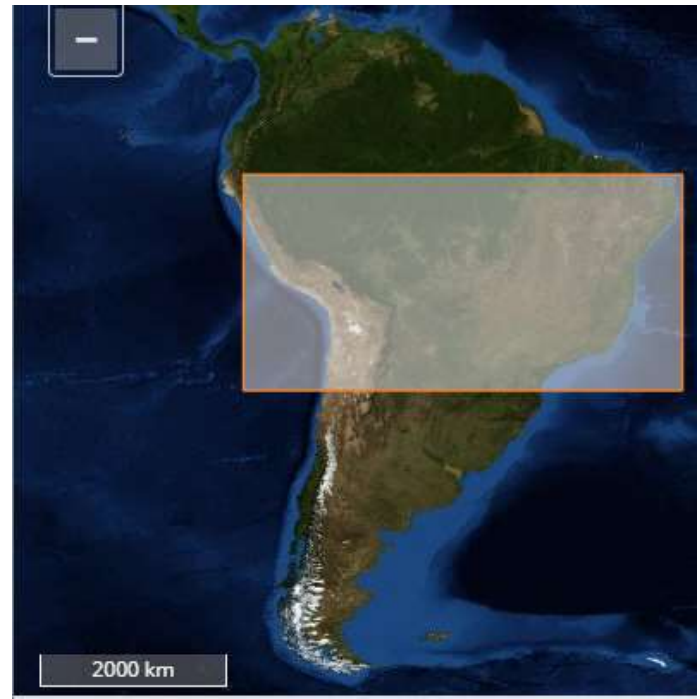

Figura 1. Área de estudio mostrado en forma rectangular para (a.) Norte de Suramérica, (b.) Centro de Suramérica. Fuente: autores.

\section{- Análisis espacio-temporal incendios entre 2009 y 2019}

Francois (2011) afirma "Se utilizó información del espectrorradiómetro de imágenes de media resolución (MODIS) a bordo de los satélites Aqua y Terra de la NASA. Esta información se obtuvo a partir de la plataforma "Fire Information for Resource Management System" (FIRMS) (https://firms.modaps.eosdis.nasa.gov/).

Se seleccionaron aquellos incendios entre 2009 y 2019 con una confianza mayor o igual al $75 \%$ con el fin de asegurar su alta calidad. Los incendios seleccionados fueron contados diariamente mediante el lenguaje de programación $R$. $R$ es el software básico de referencia en el mundo de la estadística, la herramienta más potente y eficiente del mercado, además, es un software OPEN SOURCE para todos los sistemas operativos, es decir que es de código abierto, y por tanto, no tiene costo de adquisición. Este lenguaje de programación está pensado para de cálculos estadísticos, cálculos matemáticos y creación de gráficos de una manera fácil y rápida". Gracias a los paquetes zoo y tidyverse de $R$ se pudo sistematizar el análisis espacial y temporal de los incendios. Se analizó su temporalidad a nivel mensual y anual mediante diagramas de caja y bigote con el fin de identificar los periodos más y menos críticos en el norte y centro de Suramérica. Conjuntamente se aplicó la prueba de Kruskal-Wallis con el fin de estimar si existía una diferencia significativa entre los promedios mensuales y anuales de conteos diarios de incendios para ambas zonas de estudio. Así mismo, a partir de la información proveniente de MODIS se identificaron espacialmente los incendios cuyo poder radiativo [MW] se encontró por encima del percentil 90. Las zonas del norte y centro de Suramérica más críticas para la población debido a su alto poder radiativo se identificaron paquetes de R: raster, rgdal, rgeos, stringr, sf, tidyverse, RColorBrewer, cowplot, ggpubr, ggspatial, rnaturalearth, rnaturalearthdata, ggplot2. 


\section{RESULTADOS Y DISCUSIÓN}

\section{- Análisis espacial de los incendios del norte de Suramérica}

Mendez Espinosa et al.,(2019) afirma "Durante el periodo 2009-2019, el NSA fue principalmente afectado por los incendios provenientes de la cuenca del Orinoco Colombo-Venezolano y del caribe Colombiano (ambas llanuras), en concordancia con lo obtenido por Anaya (2009) en su estudio para Colombia entre el 2001 y el 2006 en el que establece que los incendios fueron la mayor fuente de gases efecto invernadero en el país. Este impacto se generó principalmente durante los primeros meses del año. Durante estos meses, se presentó una disminución en la precipitación debido al desplazamiento de la Zona de Convergencia Intertropical (ZCIT) hacia la zona sur". Mendez Espinosa et al. (2020) afirma "Los incendios del NSA presentaron un poder radiativo con una mediana de 34,5 MW (1er cuartil: 20,7 MW; 3er cuartil: 64,2 MW) y valores máximos de hasta 3859,4 MW. La distribución de los incendios con un poder radiativo por encima del percentil 90 (> $118,9 \mathrm{MW}$ ) se muestra en la Figura 2. Su ubicación y poder radiativo preocupa ya que se ha relacionado con la mala calidad del aire en zonas circundantes".

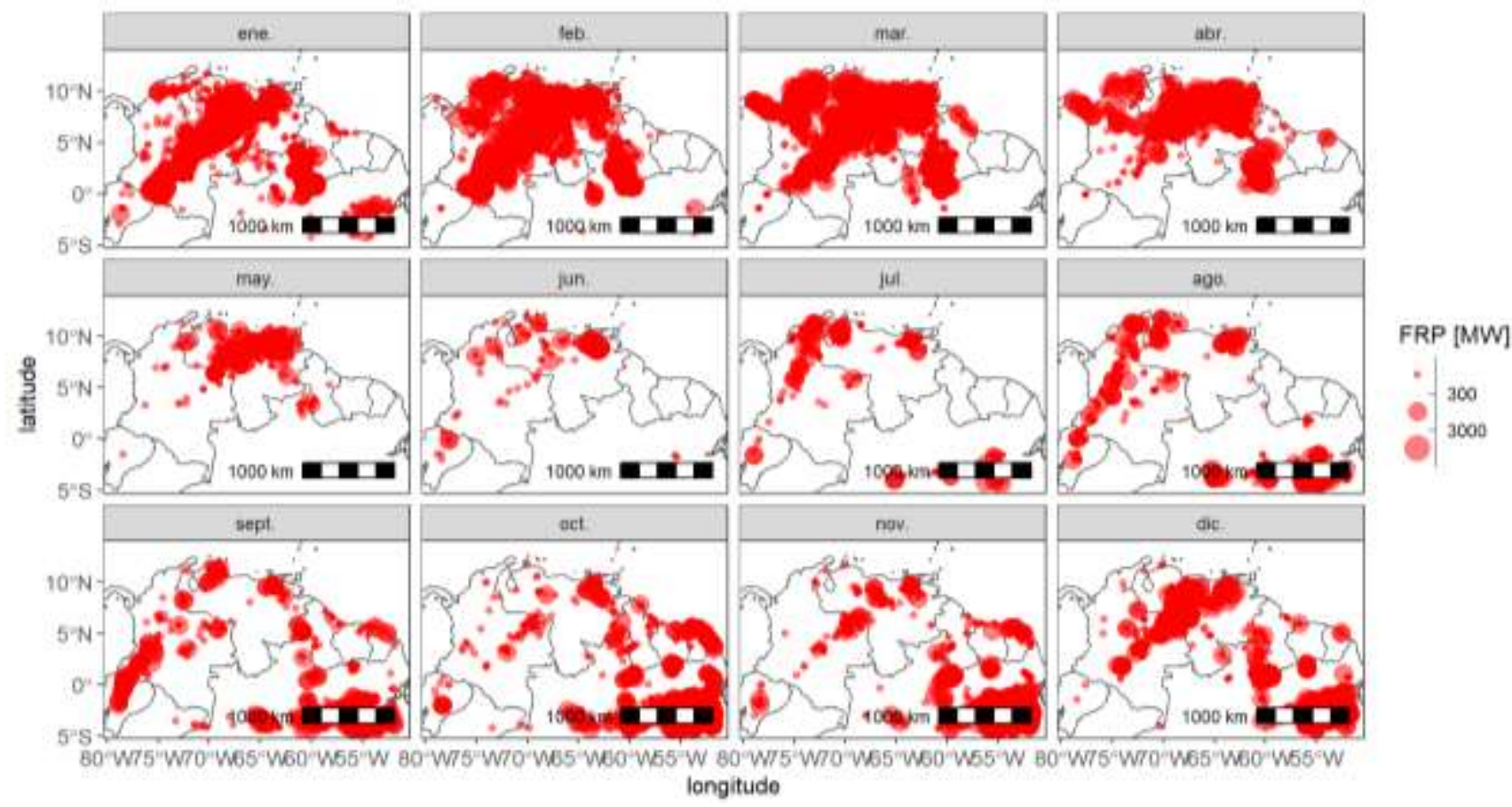

Figura 2. Distribución mensual de los incendios del norte de Suramérica con un poder radiativo [MW] por encima del percentil 90 ( $>118,9$ MW) entre 2009 y 2019. Fuente: autora

A diferencia del NSA, los incendios del CSA durante el periodo 2009-2019 se ubicaron en más del $90 \%$ de la cuenca del Amazonas, principalmente durante julio, agosto y septiembre, lo que sugiere su extenso impacto sobre la flora y fauna. Durante estos 
meses, Brasil, Perú, Bolivia, y Paraguay registran presencia de incendios en diferentes zonas de su territorio. Sin embargo, se presenta un impacto recurrente $y$ espacialmente significativo en Paraguay y Brasil (Figura 3). Los incendios del CSA presentaron un poder radiativo con una mediana de $44 \mathrm{MW}$ (1er cuartil: 24,5 MW; 3er cuartil: $89,4 \mathrm{MW}$ ) y valores máximos de hasta 13294,5 MW. La distribución de los incendios con un poder radiativo por encima del percentil 90 ( $>179,5 \mathrm{MW}$ ) se muestra en la Figura 3. Así las cosas, los incendios del CSA no solo tuvieron una mayor extensión de impacto en comparación al NSA, también tuvieron un mayor poder de destrucción debido a su alto poder radiativo.

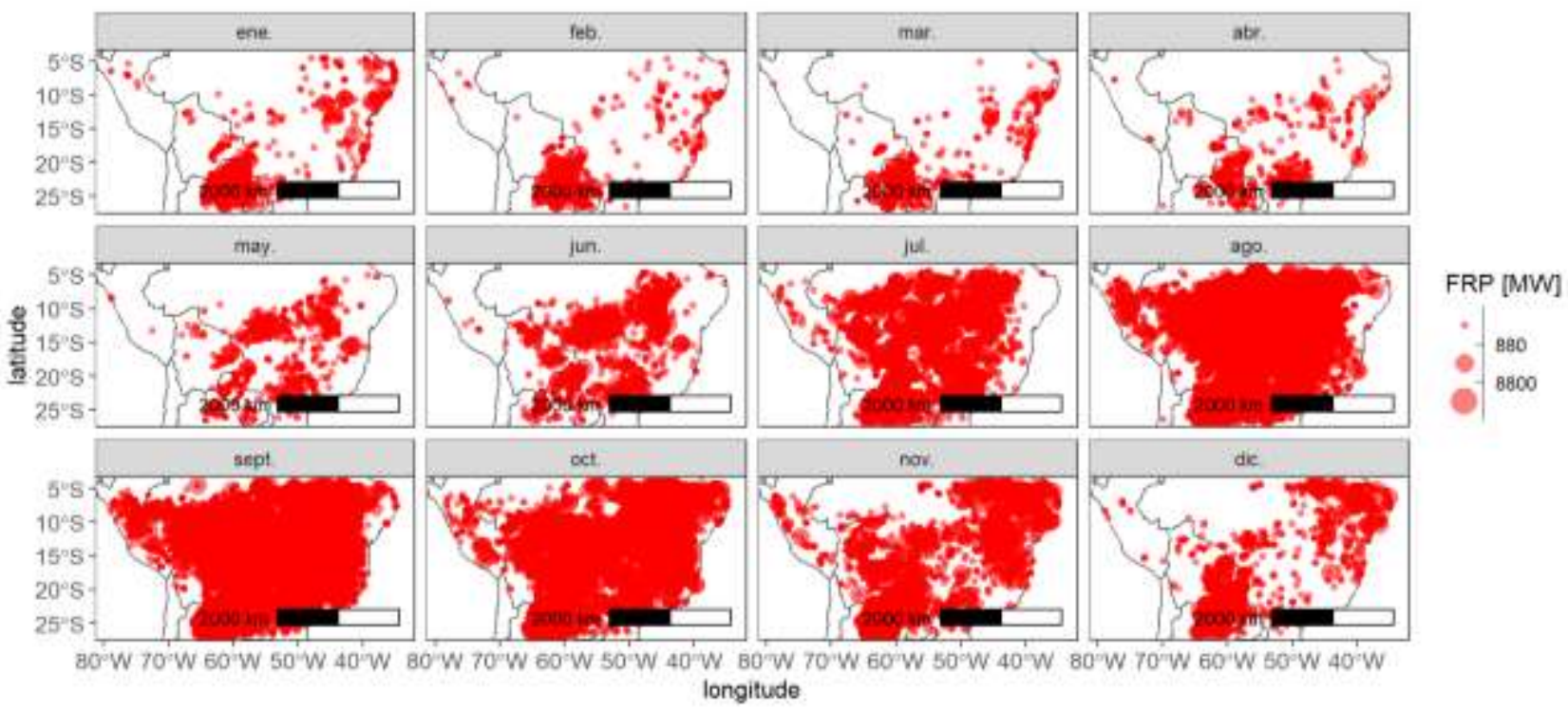

Figura 3. Distribución mensual de los incendios del centro de Suramérica con un poder radiativo [MW] por encima del percentil 90 (> 179,5 MW) entre 2009 y 2019. Fuente: autora

\section{- Análisis temporal de los incendios del norte de Suramérica}

En el NSA, durante el 2009-2019 el conteo diario de incendios presenta una mediana de 61 incendios/día (1er cuartil: 27; 3er cuartil: 130) y valores máximos de hasta 1460 incendios/día. El promedio de 106,4 incendios/día sugiere una mayor concentración de días con gran número de incendios (> 61). Por otro lado, en el CSA, durante el 2009-2019 el conteo diario de incendios presenta una mediana con 83 incendios/día más que el NSA (1er cuartil: 57; 3er cuartil: 501) y valores máximos de hasta 7579 incendios/día (6119 incendios/día más que en el NSA). El promedio de 487,6 incendios/día sugiere una mayor concentración de días con gran número de incendios ( $>144$ ).

Los resultados anuales del conteo diario de incendios entre 2009-2019 muestran que el CSA pudo llegar a presentar 5000 incendios más que el NSA. Ambas zonas presentaron altas anomalías durante el 2010 y 2019 (Figura 4). Sin embargo, a pesar de estar en una misma región (Latinoamérica) presentan un comportamiento diferenciado. 
En el NSA se presume una tendencia creciente alta en el número diario de incendios, mientras que en el CSA la tendencia ascendente es menos pronunciada. A nivel mensual (Figura 5), el NSA se ve principalmente impactado durante los primeros cuatro meses del año, mientras que el CSA presenta un incremento en más del $100 \%$ entre julio y noviembre en comparación con los demás meses. Al comparar los grupos a nivel anual y mensual para cada zona según la prueba de KruskalWallis se obtuvo una diferencia significativa en todos los casos (valor-p < 1\%).

(a.) NSA

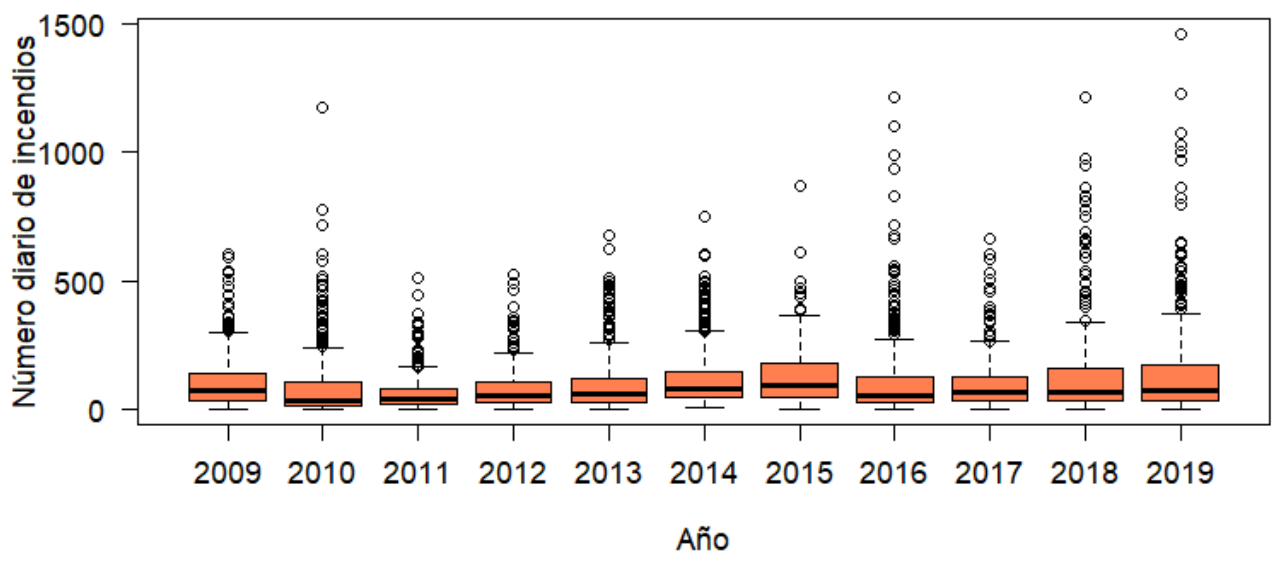

(b.) CSA

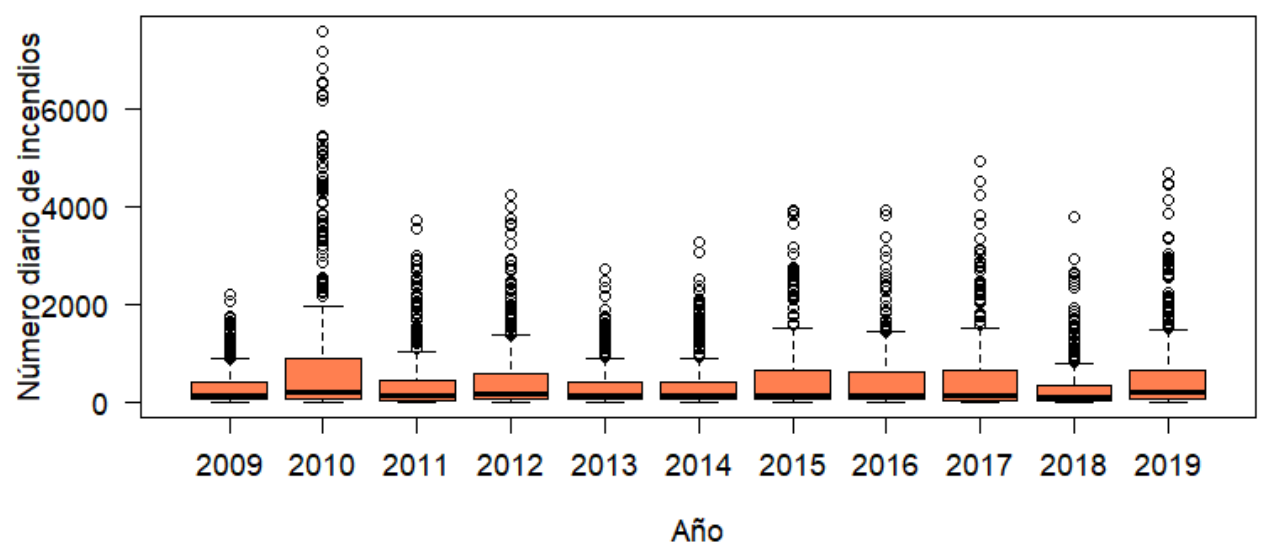

Figura 4. Diagrama anual de caja y bigotes asociado al conteo diario de incendios en el (a.) norte de Suramérica y (b.) centro de Suramérica durante 2009-2019. Fuente: autora 
(a.) NSA

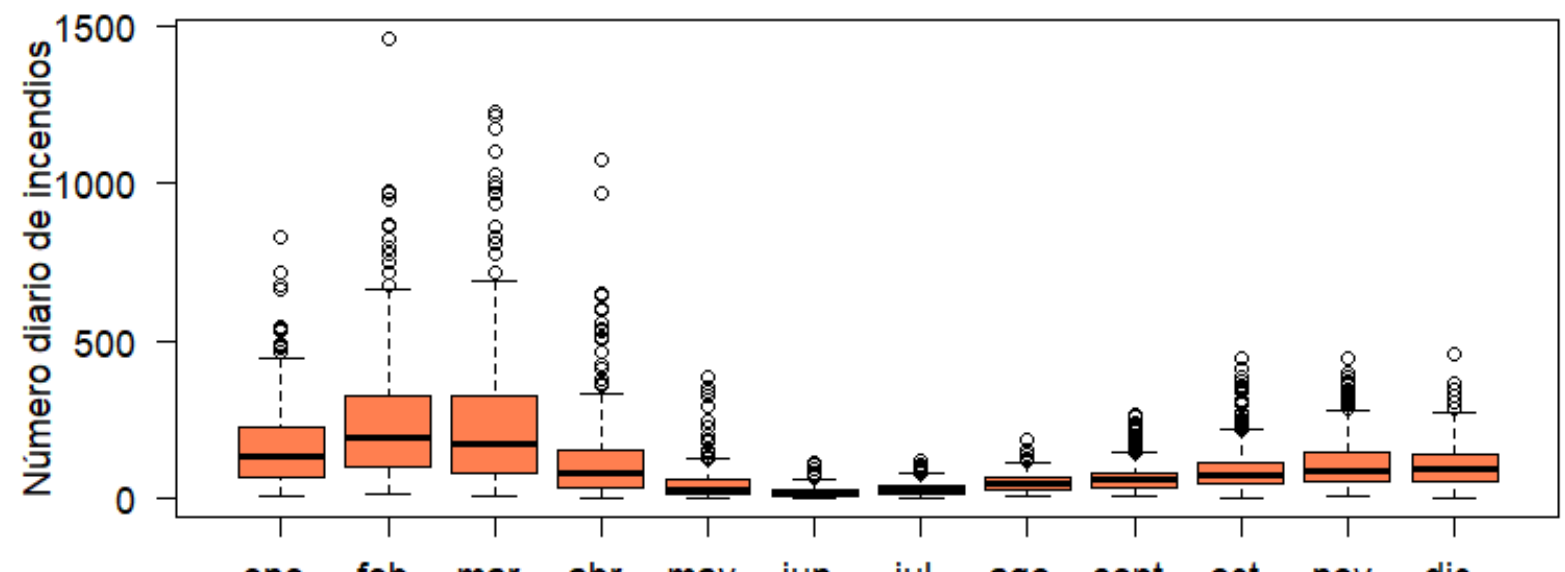

ene. feb. mar. abr. may. jun. jul. ago. sept. oct. nov. dic.

(b.) CSA

Mes

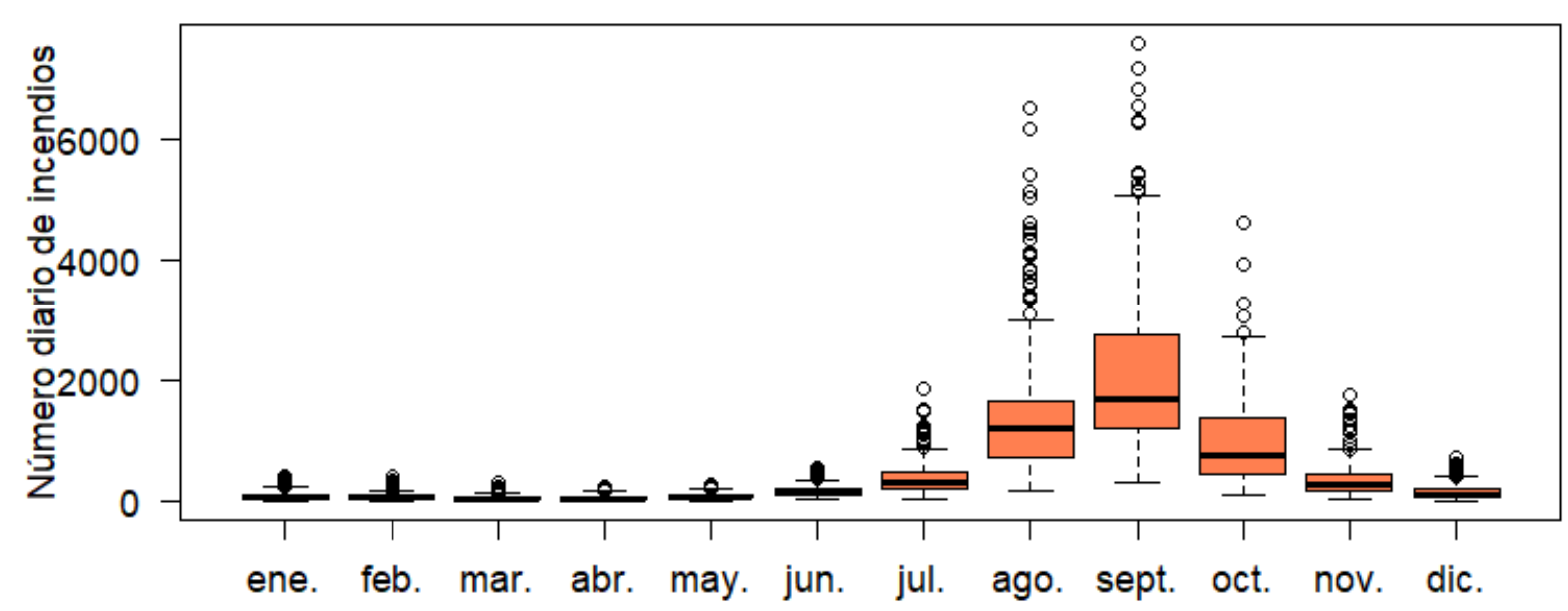

Mes

Figura 5. Diagrama multimensual de caja y bigotes asociado al conteo diario de incendios en el (a.) norte de Suramérica y (b.) centro de Suramérica durante 2009-2019. Fuente: autora

La comparación de los resultados obtenidos en esta investigación con los resultados obtenidos por Armenteras en su estudio "Caracterización de las interacciones del patrón espacial del fuego con el clima y la vegetación en Colombia" evidencia que también la mayor incidencia fue en el año 2003 donde el número de incendios incremento generando un impacto en más de
1.000.000 hectáreas. Así mismo, se evidencio una semejanza con el incremento después del año 2010 respecto a la cantidad de hectáreas afectadas e incendios provocados.

Al comparar los resultados de la presente investigación con el artículo científico "Dinámica e impulsores de la deforestación en diferentes tipos de bosques en América 
Latina: tres décadas de estudios (19802010)" por Armenteras se constató las pérdidas en biodiversidad por incendios generados en los países del NSA. Conjuntamente se identificó que la deforestación y los incendios forestales incrementaron anualmente, especialmente por influencia antrópica.

En la investigación "Dinámica del paisaje en la Amazonía noroccidental: una evaluación de pastos, incendios y cultivos ilícitos como impulsores de la deforestación tropical" de Armenteras, se infirió que durante 2009 (primer año de estudio en esta investigación) el noroeste de la Amazonia colombiana presento un desequilibro en sus ecosistemas debido a la intencionalidad del uso del fuego. Se estimo un aumento anual del $0,06 \%$ en la deforestación en la que la quema fue uno de los factores principales durante el periodo comprendido entre 2000 y 2009. En la presente investigación no se encontró una tendencia ascendente en el número de incendios diarios por encima del $1 \%$, sin embargo, si se pudo evidenciar cambios anuales por posibles influencias de los fenómenos ENOS: el Niño y la Niña.

Los incendios forestales relacionados con las prácticas de tala y quema, tradicionalmente utilizados para la conversión de bosques en tierras agrícolas y pastizales, se han destacado como un factor importante que degrada y erosiona los bosques y altera su composición y estructura de acuerdo con las investigaciones realizadas por Armenteras en el 2013. A su vez, la tala de bosques también da como resultado la fragmentación del bosque, lo que aumenta el número de bordes de bosque $y$, por lo tanto, la alteración de las condiciones de humedad del sub-dosel, lo que a su vez aumenta la susceptibilidad de los fragmentos restantes al fuego
(Armenteras, 2013). Así las cosas, el uso del fuego para la limpieza y el manejo de la tierra son una de las principales amenazas para los bosques neotropicales y una de las fuentes más importantes de emisiones de contaminantes climáticos y atmosféricos.

\section{CONCLUSIONES}

Se identificó que en el NSA los focos de incendios aumentan en los primeros 3 meses de cada año; mientras que en el CSA se presenta entre Julio-noviembre.

EI NSA es afectado por incendios de más de 3000 MW en la cuenca de la Orinoquia; mientras que el CSA es afectado en más de un $90 \%$ por incendios de más 8800 MW.

Los incendios del CSA no solo tuvieron una mayor extensión de impacto en comparación al NSA, también tuvieron un mayor poder de destrucción debido a su alto poder radiativo.

\section{CONTRIBUCIÓN DE LA AUTORÍA}

López-Guevara B.: Investigación, escritura, borrador original, análisis de datos.

\section{AGRADECIMIENTOS}

La autora agradece a la NASA por la identificación de las anomalías térmicas, y a los creadores de $\mathrm{R}$ Project. Así mismo, agradece la asesoría y dirección del docente Juan Felipe Mendez Espinosa de la ECAPMA de la Universidad Nacional Abierta y a Distancia.

\section{REFERENCIAS}

Alencar, A. C., Solorzano, L. A., \& Nepstad, D. C. (2004). MODELADO DE INCENDIOS FORESTALES EN UN PAISAJE DEL AMAZÓNICO ORIENTAL. Ecological 
Applications, 14(0), 139-149.

https://doi.org/https://doi.org/10.1890/01$\underline{6029}$

Armenteras, D., Rodríguez, N., \& Retana, J. (2013). Landscape Dynamics in Northwestern Amazonia: An Assessment of Pastures, Fire and Illicit Crops as Drivers of Tropical Deforestation. In Plos One (Vol. 8, Issue 0). https://doi.org/https://doi.org/10.1371/journ al.pone.0054310

Francois, J. (2011). Aplicaciones del sensor MODIS - CIGA-UNAM.

Marengo, J. A., Nobre, C. A., Tomasella, J., Oyama, M. D., Sampaio de Oliveira, G., Oliveira, R. de, Camargo, H., Alves, L. M., \& Foster Brown, I. (2008). The Drought of Amazonia in 2005. AMS, 21(0), 495-516. https://doi.org/https://doi.org/10.1175/2007 JCLI1600.1

Mendez Espinosa, J. F., Belalcazar, L. C., \& Morales, B. (2019). Regional air quality impact of northern South America biomass burning emission. Sciencedirect, 203(0), $131-140$. https://doi.org/https://doi.org/10.1016/j.atm osenv.2019.01.042

Mendez Espinosa, J. F., Rojas, N. Y., Vargas, J., Pachón, J. E., Belalcazar, L. C., \& Ramírez, O. (2020). Air quality variations in Northern South America during the COVID-19 lockdown. Sciencedirect, 749(0). https://doi.org/https://doi.org/10.1016/j.scit otenv.2020.141621
Morton, D. C., Defries, R. S., Randerson, J. T., Giglio, L., Schroeder, W., \& Van Der Werf, G. R. (2008). Agricultural intensification increases deforestation fire activity in Amazonia. Wiley Online Library, 14(0), Pages 2262-2275.

https://doi.org/https://doi.org/10.1111/j.136 5-2486.2008.01652.x

Müller, D., Suess, S., Hoffmann, A. A., \& Buchholz, G. (2013). The Value of SatelliteBased Active Fire Data for Monitoring, Reporting and Verification of REDD+ in the Lao PDR. Springer King, 7-13. https://doi.org/10.1007/ s10745-013-9565$\underline{0}$

Parr, C. L., \& Chown, S. L. (2003). Burning issues for conservation: A critique of faunal fire research in Southern Africa. Austral Ecology, 28(0), 384-395. https://doi.org/https://doi.org/10.1046/j.144 2-9993.2003.01296.x

Pimm, S. L., Russell, G. J., Gittleman, J. L., \& Brooks, T. M. (1995). The Future of Biodiversity. Science, 269(0), 347-350. https://doi.org/10.1126/science.269.5222.34 7

Ray, D., Nepstad, D., \& Moutinho, P. (2005). MICROMETEOROLOGICAL AND CANOPY CONTROLS OF FIRE SUSCEPTIBILITY IN A FORESTED AMAZON LANDSCAPE. Ecological Applications, 15(0), 1664-1678. https://doi.org/https://doi.org/10.1890/05$\underline{0404}$

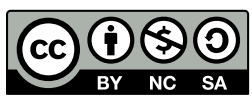

Licencia de Creative Commons

Revista Agricolae \& Habitat is licensed under a Creative Commons Reconocimiento-NoComercial-CompartirIgual 4.0 Internacional License. 